\title{
VARIAÇÃO RADIAL DA MASSA ESPECÍFICA BÁSICA DA MADEIRA DE Eucalyptus grandis W. Hill ex Maiden
}

\author{
Rômulo Trevisan*, Clóvis Roberto Haselein**, Rafael Rodolfo de Melo***, \\ Diego Martins Stangerlin***, Rafael Beltrame****, Darci Alberto Gatto*****, Leandro Calegari****** \\ *Eng. Florestal, M.Sc., UNOESC - romulo_trevisan@yahoo.com.br \\ **Eng. Florestal, Ph.D., UFSM - haseleic@ccr.ufsm.br \\ ***Eng. Florestal, Mestrando em Eng. Florestal, UFSM - rrmelo2@yahoo.com.br - diego_stangerlin@yahoo.com.br \\ ****Graduando em Engenharia Florestal, UFSM - browbeltrame@yahoo.com.br \\ *****Eng. Florestal, Dr., UFPel - darcigatto@yahoo.com \\ ******Eng. Florestal, M.Sc., Doutorando em Ciências Florestais, UFV - leandrocalegari@yahoo.com.br \\ Recebido para publicação: 25/05/2007 - Aceito para publicação: 11/02/2008
}

\begin{abstract}
Resumo
Este estudo teve como objetivo verificar a variação radial da massa específica básica da madeira Eucalyptus grandis W. Hill ex Maiden, aos 14 anos de idade, proveniente de povoamentos situados próximos ao litoral norte do Rio Grande do Sul. Selecionaram-se 32 árvores com base nos diâmetros de árvores dominantes e médias, e após a derrubada, retirou-se um disco à altura do peito (DAP), destinado à determinação da massa específica básica na posição radial. Os resultados evidenciaram que a massa específica básica, tanto para árvores dominantes quanto para médias, apresentou valores mais baixos na região da medula $\left(0,37 \mathrm{~g} / \mathrm{cm}^{3}\right)$, seguida de acréscimo até a região mais periférica do tronco $(0,43$ $\mathrm{g} / \mathrm{cm}^{3}$ ). Não foram encontradas diferenças de massa específica entre madeiras de árvores médias e dominantes.
\end{abstract}

Palavras-chave: Eucalyptus grandis; massa específica básica; qualidade da madeira.

\begin{abstract}
Radial Variation of basic density of the wood of Eucalyptus grandis W. Hill ex Maiden. This study was undertaken to determine the radial variation of basic density of the wood of 14 years old Eucalyptus grandis W. Hill ex Maiden stands, located in the northern coast of Rio Grande do Sul. Thirty two trees were selected on the basis of the dominant and average diameter, and after cutting down, one disk was taken at breast height (DBH), for basic density determination in the radial direction. The results indicated that the basic density, both for dominant and average trees, presented lower values at the pith region $\left(0,37 \mathrm{~g} / \mathrm{cm}^{3}\right)$ increasing until the more peripheral region of the trunk $\left(0,43 \mathrm{~g} / \mathrm{cm}^{3}\right)$. No differences in basic density between dominant and average diameter trees were observed.

Keywords: Eucalyptus grandis; basic density; wood quality.
\end{abstract}

\section{INTRODUÇÃO}

As restrições impostas à utilização de madeiras nobres, devido ao uso indiscriminado e insustentável dessa matéria-prima e à crescente preocupação com a conservação das espécies nativas produtoras de madeira, foram quesitos importantes para o êxito do gênero Eucalyptus no setor florestal brasileiro. Desde então, a busca por produtividade e qualidade desses povoamentos florestais tem sido alvo de inúmeras pesquisas.

A correta utilização de um material está associada às suas características físico-mecânicas. A madeira não foge à regra, tornando-se importante o conhecimento de suas variações, a fim de se prognosticar seu comportamento em diferentes utilizações (PAES et al., 1995; LOPES, 2003; 
TRUGILHO et al., 2007). A massa específica é uma das propriedades que mais fornece informações sobre as características da madeira, por estar relacionada com sua resistência e rigidez. Apresenta-se em função da parede celular, dimensões, tipos de células e, em menor proporção, da quantidade de componentes extrativos presentes por unidade de volume (PANSHIN; DE ZEEUW, 1980; HAYGREEN; BOWYER, 1982).

Conforme Kollmann; Côté (1968), a massa específica da madeira pode ser alterada em função das características da própria espécie, bem como das influências externas, assim como das variações ambientais e intervenções silviculturais. As causas da alteração desse parâmetro tecnológico podem ser indiretas, ou melhor, podem ocorrer pela modificação de outras características da própria madeira, em detrimento da espécie ou, então, pela influência do meio ambiente em que ela se desenvolve.

De acordo com Bodig; Jayne (1982), podem ocorrer, ainda, variações significativas na massa específica básica da madeira, principalmente entre gêneros, espécies do mesmo gênero e, até mesmo, entre diferentes partes da mesma árvore.

Para Panshin; De Zeeuw (1980) e Lima et al. (2000), a massa específica aumenta muito rapidamente durante o período juvenil, cresce mais lentamente numa fase intermediária da idade, até se tornar mais ou menos constante na fase da maturidade da árvore.

Segundo Tomazello Filho (1985), Silva et al. (2004) e Alzate et al. (2005), as variações da massa específica nos sentidos longitudinal e radial para a madeira de Eucalyptus estão relacionadas com a idade da árvore, a amostragem, o genótipo e as condições ambientais. Com relação ao sentido radial, uma tendência bem definida de aumento dessa característica tecnológica tem sido observada e foi confirmada pelos resultados obtidos por Malan (1995), Carmo (1996), Oliveira (1998), Lima et al. (2000), Oliveira; Silva (2003) e Silva et al. (2004).

Este trabalho foi desenvolvido com árvores dominantes e médias, com o objetivo de verificar a variação radial da massa específica básica da madeira de Eucalyptus grandis W. Hill ex Maiden.

\section{MATERIAL E MÉTODOS}

\section{Características do experimento}

O material utilizado neste estudo é proveniente de um povoamento localizado próximo ao litoral norte do estado do Rio Grande do Sul, com espaçamento inicial de 3,0 x 1,7 m, aos 14 anos de idade.

A amostragem das árvores foi realizada com base no diâmetro à altura do peito (DAP). Foram coletadas 16 árvores dominantes, com diâmetro médio de $37,1 \pm 2,1 \mathrm{~cm}$, e 16 árvores médias, com diâmetro médio de $27,0 \pm 5,5 \mathrm{~cm}$, totalizando 32 árvores.

\section{Determinação da massa específica básica}

Para o estudo dessa característica tecnológica na posição radial, retirou-se um disco, com aproximadamente dois centímetros de espessura, das árvores dominantes e médias na posição do DAP. Esses discos foram acondicionados em sacos plásticos e transportados para o Laboratório de Produtos Florestais (LPF) da Universidade Federal de Santa Maria (UFSM), onde foram marcados e seccionados em baguetas de $3 \mathrm{~cm}$ de espessura (Figura 1). Após a confecção das baguetas, foi realizada a identificação dos corpos-de-prova, com dimensões de $3,0 \times 3,0 \times 2,0 \mathrm{~cm}$ (radial, tangencial e longitudinal, respectivamente), ao longo do sentido medula-casca. Em seguida, os corpos-de-prova foram submersos em água, onde permaneceram até atingir peso constante, ou seja, completa saturação das fibras.

A massa específica básica de cada corpo-de-prova foi obtida de acordo com a norma ASTM (1997). O cálculo foi realizado através da Equação 1.

$$
M E_{b}=\frac{P o}{V u}
$$

Em que:

$\mathrm{ME}_{\mathrm{b}}=$ massa específica básica, em $\mathrm{g} / \mathrm{cm}^{3}$;

$\mathrm{Po}=$ peso seco em estufa $\left(103{ }^{\circ} \mathrm{C}\right), \mathrm{em} \mathrm{g}$;

$\mathrm{Vu}=$ volume saturado, $\mathrm{em} \mathrm{cm}^{3}$. 


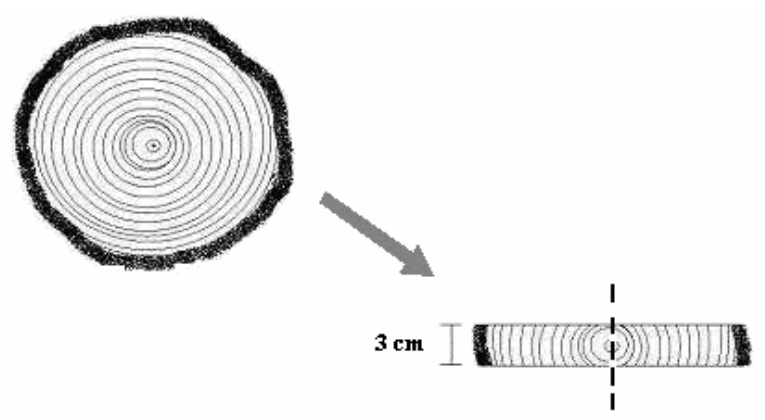

Figura 1. Ilustração do corte transversal e baguetas utilizadas na análise da massa específica básica na posição radial.

Figure 1. Illustration of cross section and increment core utilized for basic density in the radial position.

\section{Análise estatística dos dados}

Para o estudo da variação da massa específica básica no sentido radial, os dados das árvores dominantes e médias foram submetidos à análise de regressão, utilizando-se o software "Statistical Analysis System" (SAS, 1993), com o procedimento Stepwise de modelagem de regressão. O modelo máximo de regressão foi definido pela Equação 2.

$$
M E_{b}=\mathrm{f}\left(P ; \sqrt{P} ; P^{2} ; \log (P) ; 1 / P\right)
$$

Em que:

$\mathrm{ME}_{\mathrm{b}}=$ massa específica básica, em $\mathrm{g} / \mathrm{cm}^{3}$;

$\mathrm{P}=$ Posição relativa no sentido radial, em \%.

O melhor modelo foi selecionado com base nas estatísticas de $r^{2}{ }_{\text {j. }}$ (coeficiente de determinação

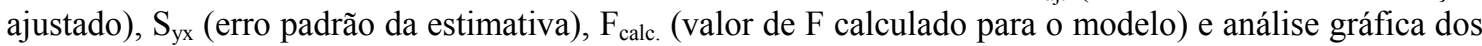
resíduos.

No modelo selecionado, foram adicionadas variáveis Dummy, definidas pelos estratos dominante e médio amostrados, e pela interação entre estratos e posição relativa (D1 = árvores dominantes; D2 = árvores médias; D3 = árvores dominantes*posição relativa e D4 = árvores médias*posição relativa). Esse método de análise verificou a possibilidade dos modelos de massa específica básica das árvores dominantes e médias serem diferentes.

$$
M E_{b}=f(X, D, D * X)
$$

Em que:

$\mathrm{ME}_{\mathrm{b}}=$ massa específica básica, em $\mathrm{g} / \mathrm{cm}^{3}$;

$\mathrm{X}=$ posição relativa no sentido medula-casca, em $\%$;

$\mathrm{D}=$ estratos;

$\mathrm{D}^{*} \mathrm{X}^{\prime}=$ interação estratos (Dummy)*X

\section{RESULTADOS E DISCUSSÃO}

Na tabela 1, são apresentados os valores médios de massa específica básica no sentido medulacasca. Observa-se um comportamento semelhante tanto para árvores dominantes como para as médias, com valores crescentes em direção à casca, à exceção da faixa compreendida entre 25 a $50 \%$ do raio, a qual apresentou uma pequena diminuição dessa característica. 
Tabela 1. Valores médios da massa específica básica de árvores dominantes e médias de Eucalyptus grandis, no sentido medula-casca, aos 14 anos de idade.

Table 1. Average basic density values of dominant and average trees of Eucalyptus grandis, from pith to bark, at 14 years of age.

\begin{tabular}{lcc}
\hline Posição & \multicolumn{2}{c}{ Massa específica básica $\left(\mathbf{g} / \mathbf{c m}^{\mathbf{3}}\right)$} \\
\cline { 2 - 3 } Medula-Casca $(\%)$ & Árvores dominantes & Árvores médias \\
\hline $0-25$ & 0,37 & 0,37 \\
$25-50$ & 0,36 & 0,36 \\
$50-75$ & 0,40 & 0,39 \\
$75-100$ & 0,43 & 0,43 \\
\hline Média geral & 0,39 & 0,39 \\
CV $(\%)$ & 9,8 & 11,8 \\
\hline
\end{tabular}

A espécie apresentou uma média aritmética de massa específica básica de $0,39 \mathrm{~g} / \mathrm{cm}^{3}$ tanto para as árvores dominantes como para as médias (Tabela 1). Esses valores estão em conformidade com os resultados obtidos por Busnardo et al. (1987) e Ferreira (1996), respectivamente, $0,41 \mathrm{~g} / \mathrm{cm}^{3}$ e $0,43 \mathrm{~g} / \mathrm{cm}^{3}$, porém para a madeira de Eucalyptus grandis com 8 anos de idade. Já Ciniglio (1998) e Lopes (2003), trabalhando com Eucalyptus grandis de 17 e 27 anos de idade, respectivamente, encontraram valores de massa específica básica maiores, com média de $0,57 \mathrm{~g} / \mathrm{cm}^{3}$ e $0,46 \mathrm{~g} / \mathrm{cm}^{3}$. Essa diferença pode ser atribuída à idade ou a fatores ambientais ou genéticos (TOMAZELLO FILHO, 1985; SILVA et al., 2004; ALZATE et al., 2005). Deve-se observar também que os valores médios apresentados na tabela 1 são aritméticos, não-ponderados, em função dos volumes de madeira determinados nos artigos citados.

\section{Árvores dominantes}

A equação de regressão encontrada para massa específica básica das árvores dominantes foi em função do quadrado da posição relativa. O teste de significância para os parâmetros da equação foi significativo em nível de 99,9\% de probabilidade de confiança. Para o coeficiente angular (b1), o valor de F' foi igual a 53,92, com probabilidade igual a 0,001 .

A variação da massa específica básica no sentido radial para árvores dominantes de Eucalyptus grandis pode ser observada na figura 2.

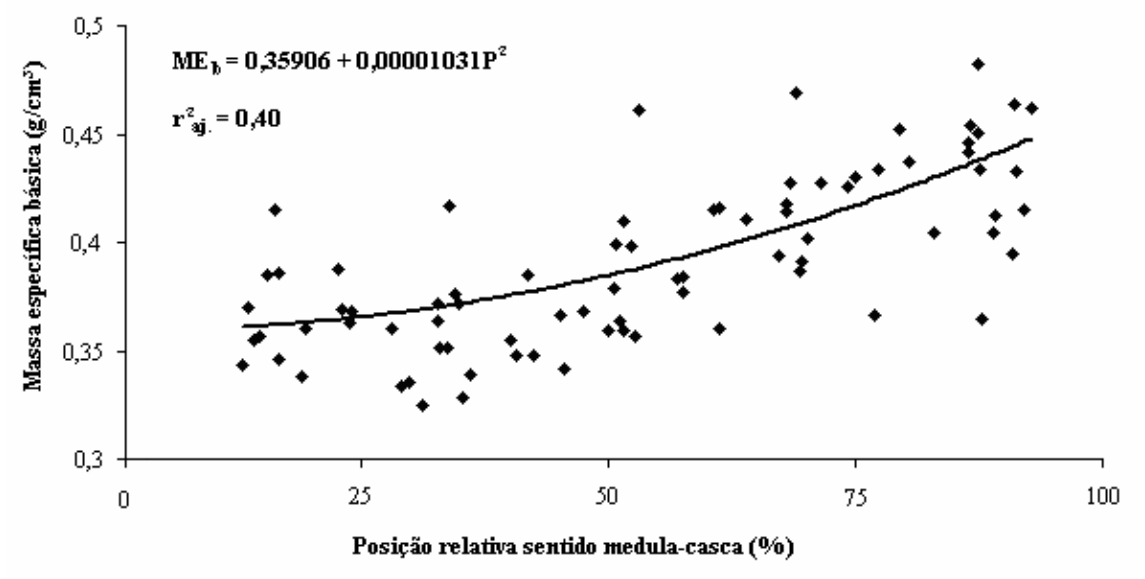

Figura 2. Variação da massa específica básica no sentido medula-casca das árvores dominantes, aos 14 anos de idade.

Figure 2. Variation of basic density from pith to bark of the dominant trees, at 14 years of age.

Observa-se que a massa específica básica, na altura do DAP, apresenta menores valores na região da medula $\left(0,37 \mathrm{~g} / \mathrm{cm}^{3}\right)$, com acréscimo até a região mais periférica do tronco $\left(0,43 \mathrm{~g} / \mathrm{cm}^{3}\right)$. 
Resultados semelhantes foram observados por Oliveira (1998) em várias espécies de eucalipto, e por Tomazello Filho (1987) para Eucalyptus globulus.

Oliveira; Silva (2003), estudando a espécie de Eucalyptus saligna, também evidenciaram um aumento dessa característica no sentido radial, com valores médios na região da medula e casca de 0,39 $\mathrm{g} / \mathrm{cm}^{3}$ e $0,55 \mathrm{~g} / \mathrm{cm}^{3}$, respectivamente.

Carmo (1996), observando as alterações da massa específica básica de seis espécies de eucalipto (Eucalyptus grandis, Eucalyptus saligna, Eucalyptus citriodora, Eucalyptus pilularis, Eucalyptus cloeziana e Eucalyptus maculata), constatou que, em todas as espécies, houve um aumento da massa específica no sentido medula-casca.

\section{Árvores médias}

A equação de regressão encontrada para a massa específica básica das árvores médias também foi em função do quadrado da posição relativa. $O$ teste de significância para os parâmetros da equação foi significativo em nível de 99,9\% de probabilidade de confiança. Para o coeficiente angular (b1), o valor de F' foi igual a 40,37, com probabilidade igual a 0,001 .

A análise da variação radial da massa específica básica para as árvores médias (Figura 3) permite afirmar que elas apresentaram o mesmo comportamento quando comparadas com as árvores dominantes, ou seja, crescente da medula até a casca. Resultados semelhantes foram observados para Eucalyptus pellita e Eucalyptus acmenioides (TOMAZELLO FILHO, 1987), e para clones desse gênero (TRUGILHO et al., 2007).

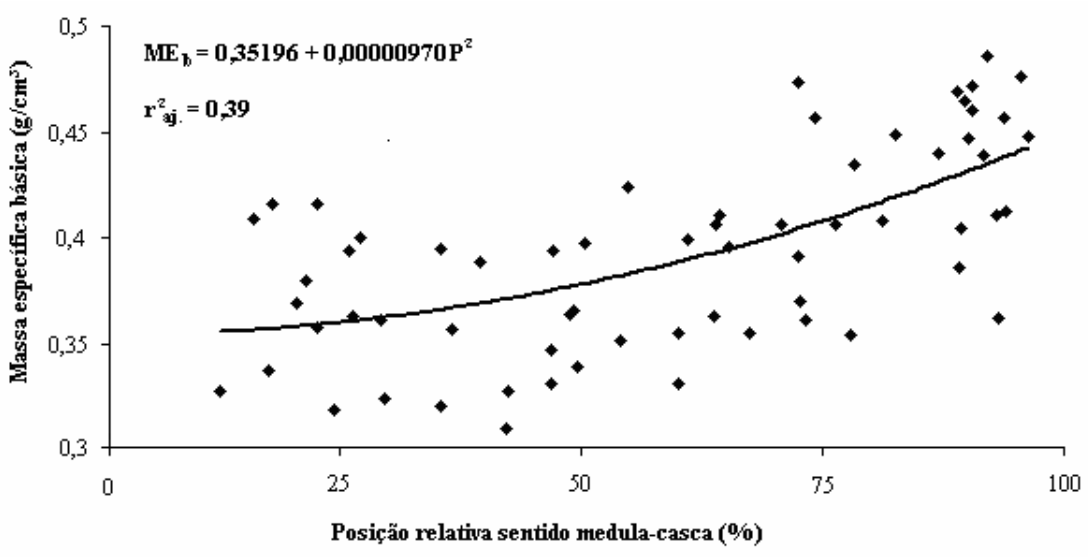

Figura 3. Variação da massa específica básica no sentido medula-casca das árvores médias, aos 14 anos de idade.

Figure 3. Variation of basic density from pith to bark of the average trees, at 14 years of age.

Brasil; Ferreira (1972), estudando a madeira de Eucalyptus grandis, e, posteriormente, Malan e Hoon (1992), afirmaram que a massa específica básica da madeira cresce no sentido medula-casca, apresentando valores médios maiores nas regiões próximas à casca. $\mathrm{O}$ aumento dessa característica, segundo os mesmos autores, foi acompanhado pelo aumento da espessura das paredes e pelo comprimento médio das fibras.

Silva (2002) e Silva et al. (2004) também verificaram uma tendência crescente da massa específica no sentido medula-casca para a madeira de Eucalyptus grandis, concluindo que os menores valores foram pertencentes às regiões próximas à medula e de idade mais reduzida, como conseqüência da presença de madeira juvenil e pelas características físico-químicas e anatômicas (MALAN, 1995).

\section{Comparação entre árvores dominantes e médias}

Para verificar se eram semelhantes os modelos que descrevem a massa específica básica, em função da posição relativa para ambos os estratos, as árvores dominantes e médias foram agrupadas e submetidas à análise de regressão com o emprego de variáveis Dummy. 
A análise estatística evidenciou que não existe diferença entre os modelos, pois todas as Dummy empregadas para verificar a influência dos diferentes estratos nessa característica (D1 com Prob. $>\mathrm{F}=0,32$ e D2 com Prob. $>\mathrm{F}=0,37$ ) não foram significativas em nível $\alpha=5 \%$ de probabilidade de erro. Dessa

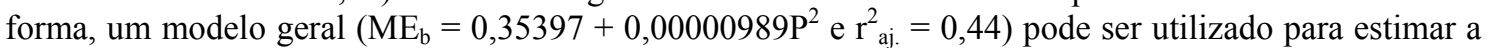
variação radial da massa específica básica tanto de árvores dominantes como de árvores médias.

\section{CONCLUSÕES}

A massa específica básica, tanto para as árvores dominantes quanto para as médias, apresentou variação semelhante no sentido medula-casca, com os menores valores dessa característica tecnológica tendo sido encontrados na região da medula, seguidos de acréscimo até a região mais periférica do tronco.

A semelhança entre os modelos de massa específica básica das árvores dominantes e médias possibilitou o ajuste de um modelo geral, que pode ser empregado para estimar essa propriedade física em ambos os estratos.

\section{AGRADECIMENTOS}

Os autores agradecem o apoio da Empresa Flosul Indústria e Comércio de Madeiras Ltda. e do Engenheiro Florestal Leonel Menezes, pela colaboração para a realização deste trabalho.

\section{REFERÊNCIAS}

ALZATE, S. B. A.; TOMAZELlO FILHO, M.; PIEDADE, S. M. S. Variação longitudinal da densidade básica da madeira de clones de Eucalyptus grandis Hill ex Maiden, E. saligna Sm. e E. grandis x urophylla. Scientia Forestalis, Piracicaba, n. 68, p. 87-95, 2005.

AMERICAN SOCIETY FOR TESTING AND MATERIALS (ASTM). D143-94: standards methods of testing small clear specimens of timber. In: Annual Book of ASTM standards. Philadelphia, 1997. p. 23-53.

BODIG, J.; JAYNE, B. Mechanics of wood and wood composites. Nova York: Van Nostrand Reinhof, 1982. 712p.

BRASIL, M. A. M.; FERREIRA, M. Variação da densidade básica e das características das fibras em Eucalyptus grandis Hill ex Maiden ao nível do dap. Revista IPEF, Piracicaba, n. 5, p. 81-90, 1972.

BUSNARDO, C. A.; GONZAGA, J. V.; FOELKEL, C. E. B.; MENOCHELli, S. Em busca da qualidade ideal da madeira do eucalipto para produção de celulose, IV. Altura ideal de amostragem para avaliação da densidade média para árvores de Eucalyptus grandis: relatório técnico. Guaíba: Riocell S. A., 1987, 18 p.

CARMO, A. P. T. Avaliação de algumas propriedades da madeira de seis espécies de eucalipto. 74 p. Dissertação (Mestrado em Ciência Florestal) - Universidade Federal de Viçosa, Viçosa, MG, 1996.

CINIGLIO, G. Avaliação da secagem de madeira serrada de Eucalyptus grandis e Eucalyptus urophylla. 73 p. Dissertação (Mestrado em Ciência Florestal) - Escola Superior de Agricultura "Luiz de Queiroz", Piracicaba, 1998.

FERREIRA, G. W. Qualidade da celulose kraft-antraquinona de Eucalyptus dunnii Maiden plantado em cinco espaçamentos em relação ao Eucalyptus grandis Hill ex-Maiden e Eucalyptus saligna Smith. 135 p. Dissertação (Mestrado em Ciências Florestais) - Universidade Federal de Santa Maria, Santa Maria, RS, 1996.

HAYGREEN, J. G.; BOWYER, J. L. Forest products and wood science: an introduction. Ames: Iowa Sate University, 1982. $459 \mathrm{p}$.

KOLlmanN, F. F. P.; CÔTÉ, W. A. Principles of wood science and technology. Berlim: SpringerVerlag, 1968. v. 1. 
LIMA, J. T.; BREESE, M. C.; CAHALAN, C. M. Variation in wood density and mechanical properties in Eucalyptus clones. In: THE FUTURE OF EUCALYPTS FOR WOOD PRODUCTS. 2000, Launceston, Tasmania. Proceedings... Launceston: IUFRO. 2000, p. 282-291.

LOPES, M. C. Agrupamento de árvores matrizes de Eucalyptus grandis em função das variáveis dendrométricas e das características tecnológicas da madeira. 93 p. Dissertação (Mestrado em Engenharia Florestal) - Universidade Federal de Santa Maria, Santa Maria, RS, 2003.

MALAN, F. S.; HOON, M. Effect of initial spacing and thinning on some wood properties of Eucalyptus grandis. South African Forestry Journal, Petroria, n. 163, p. 13-20, 1992.

MALAN, F. S. Eucalyptus improvement for lumber production. In: SEMINÁRIO INTERNACIONAL DE UTILIZAÇÃO DA MADEIRA DE EUCALIPTO PARA SERRARIA, 1., 1995, São Paulo. Anais do ... Piracicaba: IPEF/IPT, 1995. p. 1-19.

OLIVEIRA, J. T. S. Caracterização da madeira de eucalipto para a construção civil. 429 p. Tese (Doutorado) - Escola Politécnica, Universidade de São Paulo, São Paulo, 1998.

OLIVEIRA, J. T. S.; SILVA, J. C. Variação radial da retratibilidade e densidade básica da madeira de Eucalyptus saligna Sm. Revista Árvore, Viçosa, MG, v. 27, n. 3, p. 381-385, 2003.

PAES, J. B.; LIMA, C. R.; SILVA, J. M. Variação longitudinal e radial da densidade básica da madeira de algaroba (Prosopis juliflora D.C.). In: ENCONTRO BRASILEIRO EM MADEIRAS E EM ESTRUTURAS DE MADEIRA, 5., 1995, Belo Horizonte-MG. Anais... Belo Horizonte: IBRABEM, 1995. p. 225-234.

PANSHIN, A. J.; de ZEEUW, C. Text book of wood technology. 4. ed. New York: Mc Graw Hill, 1980. $722 \mathrm{p}$.

STATISTICAL ANALYSIS SYSTEM (SAS). Programa de computador, ambiente VM. Cary, Versão 6.08.1993.

SILVA, J. C. Caracterização da madeira de Eucalyptus grandis Hill ex Maiden, de diferentes idades, visando a sua utilização na indústria moveleira. 148 f. Tese (Doutorado em Ciências Florestais) - Setor de Ciências Agrárias, Universidade Federal do Paraná, Curitiba, 2002.

SILVA, J. C.; OLIVEIRA, J. T. S.; TOMAZELLO FILHO, M.; KEINERT JÚNIOR, S.; MATOS, J. L. M de. Influência da idade e da posição radial na massa específica básica da madeira de Eucalyptus grandis Hill ex. Maiden. Floresta, Curitiba, v. 34, n. 1, p. 13-22, 2004.

TOMAZELLO FILHO, M. Variação radial da densidade básica e da estrutura anatômica da madeira de Eucalyptus saligna e Eucalyptus grandis. IPEF, Piracicaba, v. 29, p. 37-45, 1985.

TOMAZELLO FILHO, M. Variação radial da densidade básica em estrutura anatômica da madeira do Eucalyptus globulus, Eucalyptus pellita e Eucalyptus acmenioides. IPEF, Piracicaba, n. 36, p. 35-42, 1987.

TRUGILHO, P. F.; BIANCHI, M. L.; ROSADO, S. C. S.; LIMA, J. T. Qualidade da madeira de clones de espécies e híbridos naturais de Eucalyptus. Scientia Forestalis, Piracicaba, n. 73, p. 55-62, 2007.

VITAL, B. R. Métodos de determinação da densidade da madeira. Viçosa: SIF/UFV, 1984. 21 p. 